Check for updates

Cite this: RSC Adv., 2019, 9, 5037

Received 5th December 2018

Accepted 25th January 2019

DOI: 10.1039/c8ra09987a

rsc.li/rsc-advances

\section{Fabrication of cefotaxime sodium-functionalized gold nanoclusters for the detection of copper ions in Chinese herbal medicines $\uparrow$}

\begin{abstract}
Cunling Ye, $\hbar^{* a}$ Yuanfei Wang, ${ }^{a}$ Shen Wang ${ }^{a}$ and Zhike Wang (iD $\hbar^{* b}$
Antibiotic-based gold nanoclusters (AuNCs) are a good sensing platform for specific recognition; however, the related studies are few. Herein, a simple and facile strategy was proposed for the fabrication of bright blue fluorescent AuNCs through the degradation product (DCTX) of cefotaxime sodium that induced the reduction of $\mathrm{HAuCl}_{4}$. Various analytical techniques were applied to characterize the prepared AuNCs@DCTX. AuNCs@DCTX exhibited a strong emission peak centered at $420 \mathrm{~nm}$ and a quantum yield of $11.8 \%$. Furthermore, an aggregation-induced fluorescence quenching mode endowed the AuNCs@DCTX probe with good specificity and sensitivity for $\mathrm{Cu}^{2+}$ detection. The proposed probe had a linear range of $0.01-40 \mu \mathrm{M}$, a precision with a relative standard deviation of $1.2 \%(n=8)$, and a detection limit of $8 \mathrm{nM}$ (signal/noise $=3$ ). Interestingly, the probe could be reused through switching the "off" and "on" states by the addition of $\mathrm{Cu}^{2+}$ and EDTA. The practicality of the sensing platform was investigated for the determination of $\mathrm{Cu}^{2+}$ in four Chinese herbal medicines (CHMs), and the results were in accordance with those obtained by the FAAS method. This study has provided an alternate way for the fabrication of thiolate-protected AuNCs for sensing applications.
\end{abstract}

\section{Introduction}

Nowadays, Chinese herbal medicines (CHMs) have gained extensive attention due to their long-standing clinical applications, reliable therapeutic efficacy and less secondary effects as compared to synthetic pharmaceuticals. ${ }^{1}$ Since CHMs readily assimilate heavy metallic elements through their roots, one of the major concerns in the usage of CHMs is the issue of safety related to the presence of some heavy metal elements, including copper $(\mathrm{Cu})$, at high concentrations. ${ }^{2,3}$ Since the toxicity and bioavailability of $\mathrm{Cu}$ in humans depend on its concentration, trace amounts of copper in CHMs should be regularly determined for the protection of public health., ${ }^{\mathbf{4 , 5}}$

The available traditional techniques, such as atomic absorption spectrometry (AAS) ${ }^{6}$ inductively coupled plasma atomic emission/mass spectrometry (ICP-AES/ICP-MS), ${ }^{7}$ capillary electrophoresis, ${ }^{2}$ and electrochemical methods, ${ }^{8}$ have been used to detect copper ions in various sample matrices. However, most of these techniques suffer from one or more limitations

${ }^{a}$ School of Chemistry and Chemical Engineering, Henan Normal University, Xinxiang, Henan 453007, China.E-mail: ycl@htu.cn

${ }^{b}$ School of Environment, Henan Key Laboratory for Environmental Pollution Control, Key Laboratory for Yellow River and Huai River Water Environment and Pollution Control, Ministry of Education, Henan Normal University, Xinxiang, Henan 453007, China.E-mail:wzk@htu.cn

$\dagger$ Electronic supplementary information (ESI) available. See DOI: 10.1039/c8ra09987a

\$ The authors contributed equally to this work. such as requirement of sophisticated and expensive instruments and well-trained operators as well as time consumption, which limit their widespread applications in routine copper ion detection. ${ }^{9}$ Moreover, CHMs have matrix effects and low copper levels that fall below the limit of quantification of many techniques. Compared to the abovementioned techniques, the fluorescence method is more favorable because of its low-cost, good selectivity, low detection limit and real-time monitoring with a fast response..$^{10-14}$

Recently, fluorescent metal nanoclusters, including gold nanoclusters (AuNCs), silver nanoclusters (AgNCs) and copper nanoclusters (CuNCs), have gained significant attention for application in analytical sensors. ${ }^{15-21}$ AuNCs, which consist of several to a few hundred $\mathrm{Au}$ atoms with a typical size less than $2 \mathrm{~nm}$, comparable to the Fermi wavelength of the electrons, have gained significant interest in chemical, biological, and environmental analysis due to their distinct advantages of simple preparation, extraordinary stability, satisfying luminescence quantum yield (QY), and biocompatibility. ${ }^{22}$ To date, some different kinds of ligands or templates (e.g., DNA, peptides, proteins, dendrimers, polymers, small thiol molecules, etc.) have been used to guide the synthesis of the AuNCs. ${ }^{15,22-24}$ Specifically, thiolate-protected AuNCs exhibit attractive features such as ultra-small hydrodynamic diameters, excellent photostability and modifiable surface properties and act as probes for the detection of $\mathrm{H}_{2} \mathrm{O}_{2}$, glucose, pyrophosphate, trypsin activity, $\mathrm{Ag}^{+}, \mathrm{Cr}^{3+}, \mathrm{Cu}^{2+}, \mathrm{Fe}^{3+}, \mathrm{Hg}^{2+}, \mathrm{Pb}^{2+}, \mathrm{NO}_{2}{ }^{-}$, and $\mathrm{S}^{2-} \cdot{ }^{25-27}$ 
As is known, some antibiotics contain a variety of functional groups, which allow them to provide multiple interactions (e.g., electrostatic, hydrophobic, dipole-dipole, $\pi-\pi$ interactions, hydrogen bonding, etc.) with the target compounds. ${ }^{28}$ Therefore, some antibiotic-type stationary phases have been developed for enantioseparation. ${ }^{29}$ This suggests that AuNCs, prepared in the presence of commercial antibiotics serving as both reductants and ligands, can have the ability to capture the analytes through multiple interactions. However, few studies have been focussed on the preparation of antibiotic-based fluorescent AuNCs for sensing applications. ${ }^{30}$ Cefotaxime sodium (CTX), the third generation of cephalosporin against Gram positive and Gram negative bacteria, ${ }^{31}$ contains nitrogen, sulphur and oxygen like many efficient ligands or templates of AuNCs. Interestingly, CTX in an alkaline medium can be entirely degraded into an equal mole of a reducing sulfhydryl compound (DCTX) in a boiling water bath. ${ }^{32}$ Thus, we believe that DCTX can be used as both a reductant and a capping ligand for the generation of DCTX-capped AuNCs (AuNCs@DCTX). Herein, water-soluble AuNCs@DCTX with a bright blue luminescence at $420 \mathrm{~nm}$ were fabricated via a facile one-pot synthesis using DCTX as the reducing and capping ligand. Furthermore, the fluorescence of the AuNCs@DCTX could be selectively quenched by $\mathrm{Cu}^{2+}$ on the basis of a mechanism involving particle aggregation-induced fluorescence quenching through the coordination of $\mathrm{Cu}^{2+}$ with $-\mathrm{NH}_{2}$ and $-\mathrm{COOH}$ on the surface of AuNCs@DCTX. Since the degree of quenching of AuNCs@DCTX is directly related to the concentration of $\mathrm{Cu}^{2+}$, we have investigated the feasibility of using this probe for the detection of $\mathrm{Cu}^{2+}$ in CHMs. Moreover, the fluorescence response could be reversibly switched between the "off" and "on" states by the addition of $\mathrm{Cu}^{2+}$ and EDTA; this clearly benefitted our understanding of the fluorescence quenching mechanisms. This study provides a new route for the synthesis of thiolate-protected AuNCs for the quantitative analysis of heavy metal ions in CHMs.

\section{Experimental}

\subsection{Reagents and chemicals}

Unless otherwise stated, all reagents were of analytical grade, obtained from commercial suppliers and used without further purification. $\mathrm{HAuCl}_{4} \cdot 4 \mathrm{H}_{2} \mathrm{O}$, cefotaxime sodium, $\mathrm{Cu}\left(\mathrm{NO}_{3}\right)_{2} \cdot 3 \mathrm{H}_{2} \mathrm{O}, \mathrm{NaCl}, \mathrm{NaOH}$ and ethylenediaminetetraacetic acid disodium salt (EDTA) were purchased from Sinopharm Chemical Reagent Co., Ltd. Other metal salts were purchased from Tianjin De'en Chemical Reagent Co., Ltd. The Pur-ALyzer $^{\mathrm{TM}}$ Mega 1000 dialysis kit $(1 \mathrm{kDa})$ was acquired from Shanghai Biotechnology Engineering Co., Ltd. All detection experiments were performed in a $0.1 \mathrm{M} \mathrm{NaH} \mathrm{NO}_{4}-\mathrm{Na}_{2} \mathrm{HPO}_{4}$ buffer $(\mathrm{pH}=7.0)$. Ultra-pure water $(18.2 \mathrm{M} \Omega)$ was used throughout the experiments.

\subsection{Apparatus}

Fluorescence spectra were obtained using the FP-6500 spectrofluorometer (JASCO, Japan). Fourier transform Infrared spectra
(FTIR) were acquired over the range $600-4000 \mathrm{~cm}^{-1}$ using the Tensor 27 FTIR spectrometer (Bruker, Germany), equipped with a germanium-attenuated total reflection (ATR) accessory. The size and morphologies of the as-synthesized samples were investigated via high-resolution transmission electron microscopy (HRTEM) using the JEOL JEM-2010 electron microscope (JEOL, Japan) at the acceleration voltage of $200 \mathrm{kV}$. Flame atomic absorption spectrometry (Perkin-Elmer, AA700) with an air-acetylene flame was used as the classical reference method to determine $\mathrm{Cu}^{2+}$ in CHMs, and the wavelength was set to $324.8 \mathrm{~nm}$ with a spectral slit-width of $0.7 \mathrm{~nm}$ and a lamp current of $15 \mathrm{~mA}$. The model T6 UV/Vis spectrophotometer (Beijing Purkinje General Instrument Co., Beijing, China) was used to obtained the absorption spectra. The $\mathrm{pH}-3 \mathrm{C}$ digital $\mathrm{pH}$ meter (Shanghai Leici Instrument Factory, Shanghai, China) was used for $\mathrm{pH}$ adjustment.

\subsection{Preparation of AuNCs@DCTX}

AuNCs@DCTX was synthesized according to the following procedure. At first, $2.5 \mathrm{mM}$ DCTX aqueous solution was obtained using a previously reported method. ${ }^{32}$ Then, DCTX (2.5 mM, $7 \mathrm{~mL}$ ) was rapidly added to boiling $\mathrm{HAuCl}_{4}(5 \mathrm{mM}, 14$ $\mathrm{mL}$ ) under vigorous stirring for $4 \mathrm{~h}$. After being cooled down to room temperature, the solution was filtered using a $0.45 \mu \mathrm{m}$ PTFE filter membrane to remove larger particles. Subsequently, AuNCs@DCTX was further purified using a dialysis membrane (MWCO: $1 \mathrm{kDa}$ ) to remove any residual unreacted species. The final AuNCs@DCTX was stored at $4{ }^{\circ} \mathrm{C}$ for further use. Herein, the concentration of the prepared AuNCs@DCTX was denoted as $1 \mathrm{X}$.

\subsection{Selectivity measurement}

To evaluate the specificity of AuNCs@DCTX for $\mathrm{Cu}^{2+}$, various competitive metal ions, including $\mathrm{Zn}^{2+}, \mathrm{Al}^{3+}, \mathrm{Ni}^{2+}, \mathrm{Co}^{2+}, \mathrm{Pb}^{2+}$, $\mathrm{Cr}^{3+}, \mathrm{Cd}^{2+}, \mathrm{Mn}^{2+}, \mathrm{Mg}^{2+}, \mathrm{K}^{+}, \mathrm{Bi}^{3+}, \mathrm{Ba}^{2+}, \mathrm{Ca}^{2+}, \mathrm{Fe}^{3+}$ and $\mathrm{Hg}^{2+}(800$ $\mu \mathrm{L}, \quad 100 \mu \mathrm{M})$, were separately mixed with $300 \mu \mathrm{L}$ of AuNCs@DCTX and $200 \mu \mathrm{L}$ of pH 7.0 phosphate buffer solution (PBS). The mixture solution was made up to $4.0 \mathrm{~mL}$ with ultrapure water and then maintained at $30^{\circ} \mathrm{C}$ for $40 \mathrm{~min}$. Moreover, the selectivity was tested by mixing the abovementioned metal ions in the presence of $\mathrm{Cu}^{2+}$ under identical conditions. The fluorescence response of AuNCs@DCTX was obtained with excitation at $321 \mathrm{~nm}$.

\subsection{Fluorescence detection of $\mathrm{Cu}^{2+}$}

For $\mathrm{Cu}^{2+}$ determination, $300 \mu \mathrm{L}$ of the prepared AuNCs@DCTX (1X) was mixed with $200 \mu \mathrm{L}$ of pH 7.0 PBS and a certain amount of $\mathrm{Cu}^{2+}$ at final concentrations ranging from $0.01 \mu \mathrm{M}$ to $40 \mu \mathrm{M}$, and the final volume was adjusted to $4.0 \mathrm{~mL}$. After incubation at $30{ }^{\circ} \mathrm{C}$ for $40 \mathrm{~min}$, the fluorescence spectra were obtained with excitation at $321 \mathrm{~nm}$ (slit width: excitation, $10 \mathrm{~nm}$; emission, 10 $\mathrm{nm})$. Then, the calibration curve for $\mathrm{Cu}^{2+}$ was constructed based on the quenched factor $\left(F_{0} / F\right)$, where $F$ and $F_{0}$ are the maximum fluorescence signals of AuNCs@DCTX in the presence and absence of $\mathrm{Cu}^{2+}$, respectively. All experiments were performed in triplicate. 


\subsection{Analysis of CHMs}

Poria, alisma, licorice and salvia were acquired from a local drugstore. A digestion method with an acid mixture of $\mathrm{HNO}_{3}$ and $\mathrm{HClO}_{4}$ was used for sample preparation. ${ }^{33}$ In brief, the four samples were washed with ultrapure water and dried at $60{ }^{\circ} \mathrm{C}$ until a constant weight was achieved. The dried samples were finely ground using an agate mortar and sieved through a 60mesh sieve. Each powder sample $(0.5 \mathrm{~g})$ was accurately weighed into a beaker with a watch-glass, followed by the addition of $10 \mathrm{~mL}$ of $\mathrm{HNO}_{3}$. The mixture was allowed to stand overnight at room temperature. The next day, after the addition of $10 \mathrm{~mL}$ of $\mathrm{HNO}_{3}$ and $5 \mathrm{~mL}$ of $\mathrm{HClO}_{4}$, the digestion was slowly carried out on an electric hot plate until the formed transparent solution was evaporated to near dryness followed by cooling at room temperature. After this, the residue was transferred to a $25.0 \mathrm{~mL}$ volumetric flask and adjusted to a nearly neutral $\mathrm{pH}$ with $2 \mathrm{M}$ $\mathrm{NaOH}$ before the volume was adjusted with ultrapure water. The resulting solution was used for the determination of $\mathrm{Cu}^{2+}$ by the proposed fluorescence method and flame atomic absorption spectrometry (FAAS).

\section{Results and discussion}

\subsection{Synthesis and characterization of AuNCs@DCTX}

AuNCs@DCTX was prepared via a one-pot synthesis, in which DCTX played a dual role as a capping agent and a reducing agent due to its numerous functional groups such as a thiol group, a heterocyclic amine, an amine group and a carboxyl group. That is, in the synthesis process, $\mathrm{Au}(\mathrm{III})$ in $\mathrm{HAuCl}_{4}$ was reduced to $\mathrm{Au}(\mathrm{I})$ by $-\mathrm{SH}$ of DCTX, followed by the coordination of $\mathrm{Au}(\mathrm{I})$ with the thiol group to form $\mathrm{Au}(\mathrm{I})$-thiolate complexes. Subsequently, a portion of $\mathrm{Au}(\mathrm{I})$ was further immediately reduced to $\mathrm{Au}(0)$ atoms by the heterocyclic nitrogen atom of DCTX in a boiling aqueous solution, and the $\mathrm{Au}(0)$ atoms were sequestrated by the $\mathrm{Au}(\mathrm{I})$-thiolate complexes. ${ }^{26,27,32,33}$ The process was very simple as $\mathrm{pH}$ adjustment and addition of other templates or reducing agents were not required. The related synthesis parameters, including the molar ratio of $\mathrm{HAuCl}_{4}$ to DCTX, the reaction temperature and the reaction time, were optimized (Fig. S1 $\dagger$ ). As shown in Fig. S1(D), $\dagger$ the obtained AuNCs@DCTX exhibited a strong fluorescence emission at $420 \mathrm{~nm}$, whereas the fluorescence of DCTX could be ignored (no peak intensity) under the same conditions; this demonstrated that the obtained fluorescence originated from the gold core of AuNCs@DCTX. To further confirm the formation of AuNCs@DCTX, the HRTEM image was obtained. Fig. 1A shows that the prepared AuNCs@DCTX has a spherical shape and uniform size distribution with an average diameter of around $2.03 \mathrm{~nm}$ (Fig. S2 $\dagger$ ). The selected area electron diffraction pattern (Fig. 1B) also provided evidence for the crystalline nature of AuNCs@DCTX. Therefore, these evidences verified that ultrasmall AuNCs@DCTX was successfully prepared using the proposed approach.

The prepared AuNCs@DCTX is inherently water soluble because some hydrophilic groups such as $-\mathrm{COOH}$ and $-\mathrm{NH}_{2}$ are included in the DCTX structures. In addition, the stability of
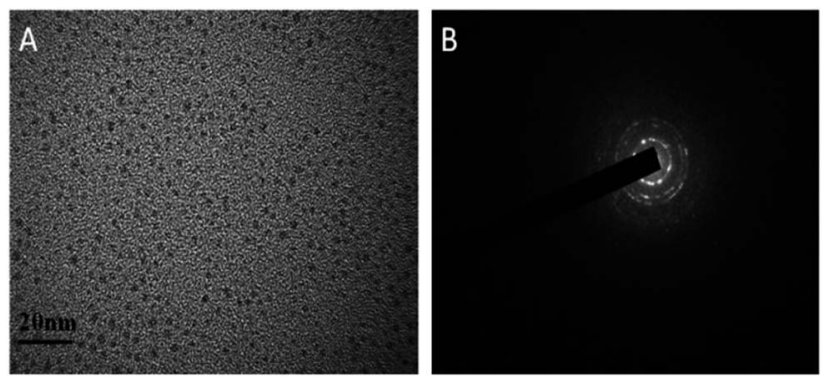

Fig. 1 An HRTEM image (A) and the selected electron diffraction pattern (B) of the prepared AuNCs@DCTX (scale bar $=20 \mathrm{~nm}$ ).

AuNCs@DCTX was systematically investigated. As shown in Fig. S3(A), $\dagger 98.9 \%$ of the initial value of the fluorescence intensity of AuNCs@DCTX was maintained after storage of AuNCs@DCTX in the dark at $4{ }^{\circ} \mathrm{C}$ for three months; this demonstrated that AuNCs@DCTX exhibited excellent storage photostability. As depicted in Fig. S3(B), $\uparrow$ there was no obvious change in the fluorescence intensity of AuNCs@DCTX in the pH range of 2.0-7.0. The fluorescence intensities of AuNCs@DCTX were not obviously affected upon varying the $\mathrm{NaCl}$ concentration from $5 \mathrm{mM}$ to $200 \mathrm{mM}$, as shown in Fig. S3(C). $\dagger$ Furthermore, Fig. S3(D) $\dagger$ displays that the fluorescence intensities of AuNCs@DCTX are preserved at $91.7 \%$ after $80 \mathrm{~min}$ of illumination at $365 \mathrm{~nm}$. In brief, these experimental results indicated that the prepared AuNCs@DCTX had ultra-high stability, providing great potential for their practical application.

Optical properties of the as-synthesized AuNCs@DCTX are presented in Fig. 2. As shown in Fig. 2A, AuNCs@DCTX has maximum excitation and emission peaks at 321 and $420 \mathrm{~nm}$, respectively. As shown in the inset of Fig.2A, the AuNCs@DCTX solution is pale yellow under visible light; on the other hand, it emits strong bright blue fluorescence under $365 \mathrm{~nm}$ UV light irradiation. Moreover, the fluorescence emission peaks showed almost no shift over a wide excitation wavelength range of 291$351 \mathrm{~nm}$ (Fig. 2B); this indicated that the observed optical signal was a real fluorescence signal from the relaxed states rather than the scattering effects. ${ }^{34}$ This may be attributed to the uniform size of AuNCs@DCTX, which is well supported by its HRTEM image. ${ }^{35}$ Fig. 2C displays the UV-vis absorption spectra of AuNCs@DCTX, $\mathrm{HAuCl}_{4}$ and DCTX, and there is no characteristic surface plasmon resonance absorption peak of gold nanoparticles near $520 \mathrm{~nm}$; this suggests that most of the particles are smaller than $2.5 \mathrm{~nm}$; this is evidence for the existence of AuNCs. ${ }^{30,36}$ The quantum yield $(\Phi)$ of AuNCs@DCTX in an aqueous solution is $11.8 \%$ at $321 \mathrm{~nm}$, with quinine sulfate (0.1 $\mathrm{mol} \mathrm{L}^{-1} \mathrm{H}_{2} \mathrm{SO}_{4}, \Phi_{\text {ref }}=0.54$ ) as the reference based on the slope method (Fig. S4†); this value is higher than those of most of the previously developed thiol-protected AuNCs. ${ }^{26,37-39}$ The stronger fluorescence of AuNCs@DCTX could be ascribed to the more electron-rich groups (e.g., $-\mathrm{NH}_{2}$ and $-\mathrm{COOH}$ ) in DCTX directly donating the delocalized electron to the gold core via surface interactions. ${ }^{40}$ Moreover, as shown in Fig. S5, $\dagger$ the fluorescence intensity of AuNCs@DCTX at $420 \mathrm{~nm}$ displayed a $69.1 \%$ decrease upon introducing $\mathrm{NaBH}_{4}$ at the concentration 

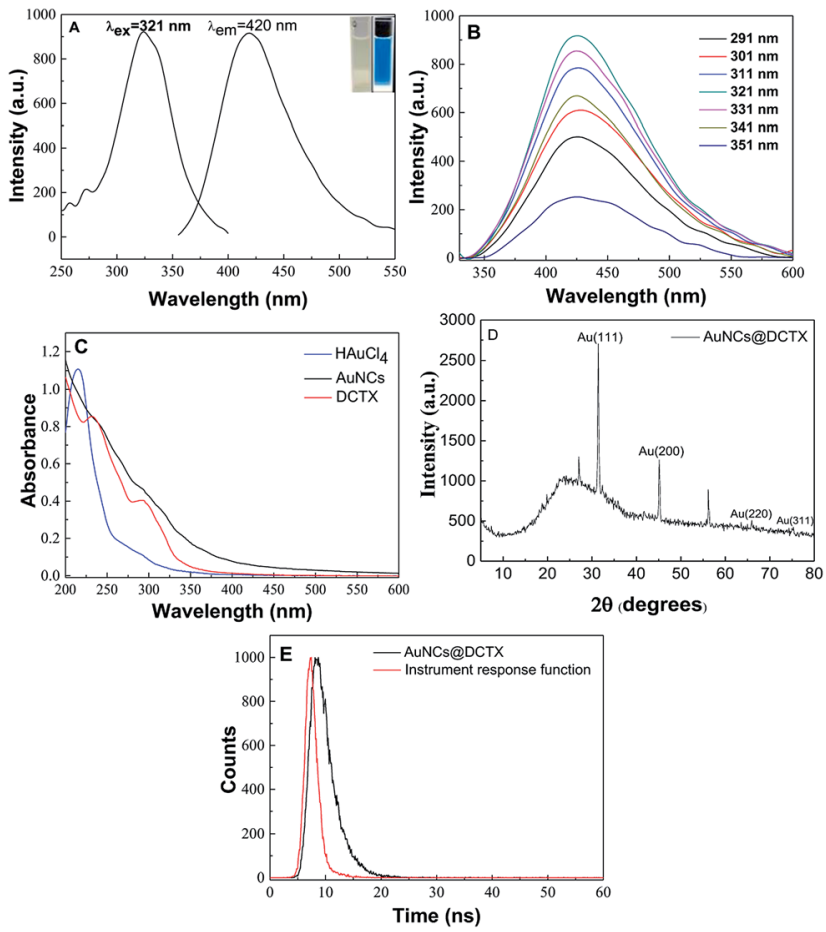

Fig. 2 Fluorescence excitation and emission spectra (A), emission spectra at different excitation wavelengths ranging from 291 to $351 \mathrm{~nm}$ (B), absorption spectra (C), XRD pattern (D) and fluorescence lifetime decay with an excitation at $321 \mathrm{~nm}$ (E) of AuNCs@DCTX. The inset in (A) shows the images of AuNCs@DCTX under (left) daylight and (right) UV lamp at $365 \mathrm{~nm}$.

of $1 \mathrm{mM}$; this indicated that the electronic structures $\mathrm{Au}(0)$ and $\mathrm{Au}(\mathrm{I})$ of $\mathrm{Au}$ coexisted in AuNCs@DCTX. ${ }^{39}$ The XRD pattern of AuNCs@DCTX in Fig. 2D demonstrates sharp peaks at $31.5^{\circ}$ and $45.1^{\circ}$ corresponding to the (111) and (200) lattice planes of AuNCs@DCTX, respectively. The other two characteristic peaks at $65.9^{\circ}$ and $75.4^{\circ}$ were labeled as the (220) and (311) diffractions of face-centered cubic (fcc) gold (JCPDS 75-2870). ${ }^{41,42}$ Fig. 2E shows the fluorescence decay profile of AuNCs@DCTX, and it can be fitted with a biexponential profile with the two components $2.380 \mathrm{~ns}$ and $6.061 \mathrm{~ns}\left(\chi^{2}=1.040\right)$. FTIR spectroscopy was also used to characterize AuNCs@DCTX. As shown in Fig. S6, $\dagger$ the peaks at $1657 \mathrm{~cm}^{-1}$ and $1562 \mathrm{~cm}^{-1}$ were attributed to the asymmetric stretching vibrations of $\mathrm{C}=\mathrm{O}$ and $\mathrm{N}-\mathrm{H}$ of DCTX, respectively; however, the DCTX-functionalized AuNCs led to a small blue shift of the corresponding peaks because of the interaction of DCTX with Au. ${ }^{41}$ Interestingly, the FTIR spectrum of AuNCs@DCTX was very similar to that of DCTX; this indicated that DCTX was successfully immobilized on the surface of AuNCs. ${ }^{43}$

\subsection{Detection of $\mathrm{Cu}^{2+}$ using AuNCs@DCTX}

To obtain a sensitive response towards $\mathrm{Cu}^{2+}$, several related factors, including the $\mathrm{pH}$, the volume of AuNCs@DCTX, the reaction temperature and the incubation time, were optimized via a univariate method. Fig. 3A shows the effect of $\mathrm{pH}$ on the relative fluorescence intensity $F_{0} / F\left(F_{0}\right.$ and $F$ refer to the
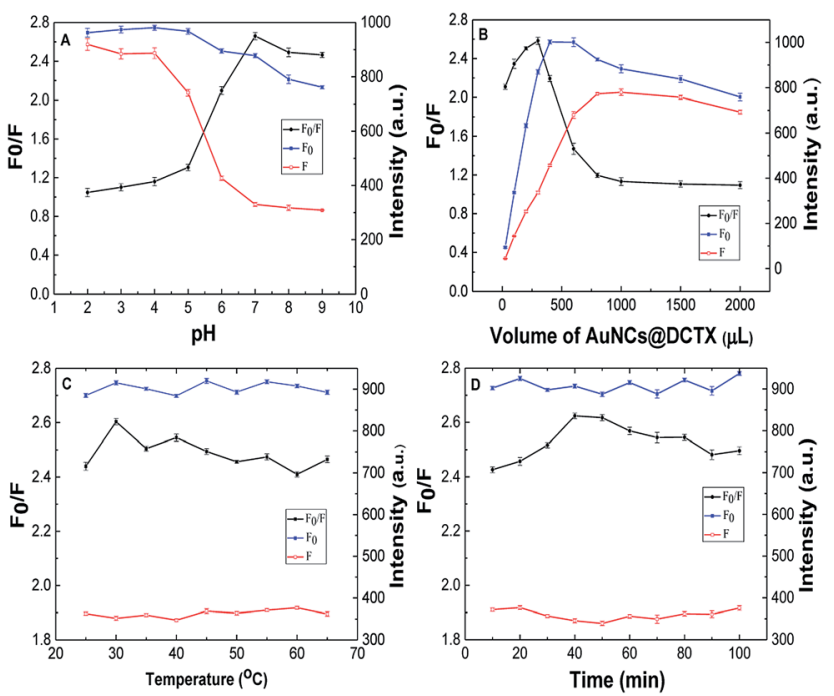

Fig. 3 The effects of $\mathrm{pH}(\mathrm{A})$, volume of AuNCs@DCTX (B), reaction temperature $(C)$ and incubation time (D) on the relative fluorescence intensity of the AuNCs@DCTX-Cu ${ }^{2+}$ system.

fluorescence intensity of AuNCs@DCTX at $420 \mathrm{~nm}$ before and after the addition of $\mathrm{Cu}^{2+}$, respectively) in PBS. It could be observed that the value of $F_{0} / F$ increased upon increasing the $\mathrm{pH}$ of the developed system from 2.0 to 7.0 and then slightly decreased in the $\mathrm{pH}$ range of 7.0-9.0. The reasons for this could be two-fold: first, a low $\mathrm{pH}$ increased the degree of amino group protonation of AuNCs@DCTX, and second, the carboxyl group of AuNCs@DCTX mainly existed as $-\mathrm{COOH}$ rather than $-\mathrm{COO}^{-}$ at a low $\mathrm{pH}$, thus weakening the complexation between $\mathrm{Cu}^{2+}$ and AuNCs@DCTX. Therefore, a pH of 7.0 was adopted for further study. The effect of the volume of AuNCs@DCTX (1X) on the fluorescence quenching efficiency is depicted in Fig. 3B. It was found that $300 \mu \mathrm{L}$ of AuNCs@DCTX was appropriate for the detection of $\mathrm{Cu}^{2+}$. The volume of AuNCs@DCTX over $300 \mu \mathrm{L}$ resulted in a low fluorescence quenching efficiency due to the self-quenching effect. However, when the volume of AuNCs@DCTX was below $300 \mu \mathrm{L}$, the limited number of fluorescence molecules in the sensor system resulted in a low fluorescence quenching efficiency. As a result, the volume of $300 \mu \mathrm{L}$ of AuNCs@DCTX was selected. Fig. 3C shows the effect of the reaction temperature on the $F_{0} / F$ value of the proposed system. The results indicated that the reaction temperature had little impact on the fluorescence quenching efficiency in the tested range from 25 to $65^{\circ} \mathrm{C}$. Considering the facile operation, a temperature of $30{ }^{\circ} \mathrm{C}$ was selected. As shown in Fig. 3D, the fluorescence quenching efficiency reached an optimum value when the reaction time was $40 \mathrm{~min}$. Thus, $40 \mathrm{~min}$ was selected as the reaction time hereinafter.

As per the design, $\mathrm{Cu}^{2+}$ can significantly affect the fluorescence of AuNCs@DCTX. Under the optimum conditions, a plot of the fluorescence quenching efficiency of AuNCs@DCTX versus the concentration of $\mathrm{Cu}^{2+}$ is presented in Fig. 4A. As depicted in Fig. 4B, a linear response between $F_{0} / F$ and $\mathrm{Cu}^{2+}$ concentration was observed over the range of $0.01-40 \mu \mathrm{M}$, and the corresponding linear equation was $F_{0} / F=0.069 C+1.028(C$ 

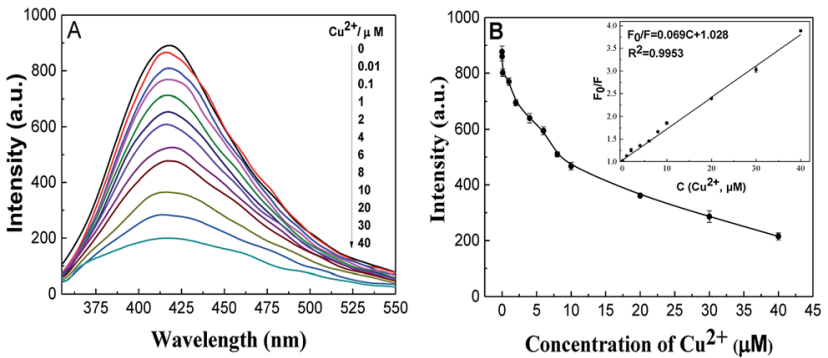

Fig. 4 Fluorescence spectra (A) of AuNCs@DCTX versus $\mathrm{Cu}^{2+}$ at different concentrations and the corresponding fluorescence responses (B) at $420 \mathrm{~nm}$ with $\mathrm{Cu}^{2+}$ at different concentrations. Inset: a linear relationship between $F_{0} / F$ and the concentration of $\mathrm{Cu}^{2+}$ ranging from 0.01 to $40 \mu \mathrm{M}$.

is the concentration of $\mathrm{Cu}^{2+}$ in $\mu \mathrm{M}$ ) with a correlation coefficient of $R^{2}=0.9953$. The relative standard deviation (RSD), determined by 8 repetitive measurements of $20 \mu \mathrm{M} \mathrm{Cu}^{2+}$, was $1.2 \%$. The limit of detection (LOD), based on a signal to noise ratio of 3 , was $8 \mathrm{nM}$, which was lower than the maximum level $(32 \mu \mathrm{M})$ of $\mathrm{Cu}^{2+}$ in drinking water defined by the World Health Organization. ${ }^{46}$ As shown in Table 1 , the performances of AuNCs@DCTX as a $\mathrm{Cu}^{2+}$ sensor were compared with those of other nanocluster-based fluorescence probes, and it was found that the proposed sensor was highly sensitive for $\mathrm{Cu}^{2+}$ detection..$^{38,39,44-50}$

The selectivity of AuNCs@DCTX towards $\mathrm{Cu}^{2+}$ was evaluated by measuring the fluorescence response of AuNCs@DCTX after adding $20 \mu \mathrm{M}$ of different common metal cations $\left(\mathrm{Zn}^{2+}, \mathrm{Al}^{3+}\right.$, $\mathrm{Ni}^{2+}, \mathrm{Co}^{2+}, \mathrm{Pb}^{2+}, \mathrm{Cr}^{3+}, \mathrm{Cd}^{2+}, \mathrm{Mn}^{2+}, \mathrm{Mg}^{2+}, \mathrm{K}^{+}, \mathrm{Bi}^{3+}, \mathrm{Ba}^{2+}, \mathrm{Ca}^{2+}, \mathrm{Fe}^{3+}$ and $\mathrm{Hg}^{2+}$ ) without and with $20 \mu \mathrm{M} \mathrm{Cu}^{2+}$, separately. Fig. 5 shows that other metal cations results in negligible or minor changes in fluorescence as compared to $\mathrm{Cu}^{2+}$; this indicates that the prepared AuNCs@DCTX probe possesses high selectivity towards $\mathrm{Cu}^{2+}$.

\subsection{Possible fluorescence quenching mechanism}

As is well-known, $\mathrm{Cu}^{2+}$ ions with paramagnetic properties are prone to quench the fluorescence of metal nanoclusters due to

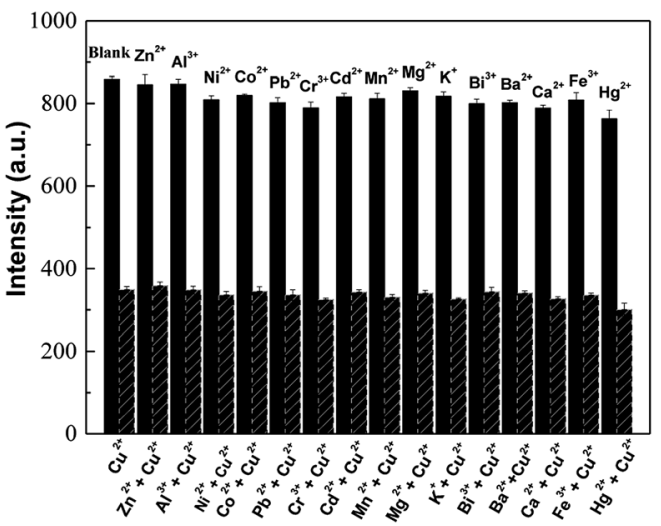

Fig. 5 Selectivity of AuNCs@DCTX towards $20 \mu \mathrm{MCu}^{2+}$ and $20 \mu \mathrm{M}$ of other common metal cations under the optimum conditions.

their strong affinity to some functional groups present on the surface of metal nanoclusters. ${ }^{39,44,51}$ We rationalize that DCTX as the capping and reducing agent contains some amino and carboxyl groups and is a high-affinity chelating agent towards $\mathrm{Cu}^{2+}$ ions like glutathione; ${ }^{45}$ thus, it would lead to fluorescence quenching of AuNCs@DCTX in the presence of $\mathrm{Cu}^{2+}$ ions. We explored the practicality of the as-prepared AuNCs@DCTX system for the detection of $\mathrm{Cu}^{2+}$. Interestingly, it was observed that the fluorescence of AuNCs@DCTX was selectively quenched by $\mathrm{Cu}^{2+}$. This phenomenon of fluorescence quenching might be due to a high possibility of aggregation of nanoclusters via complexation between the $\mathrm{Cu}^{2+}$ ions and the amino and carboxyl groups of DCTX grafted on the surface of AuNCs; this blocked the effective Au-S charge transfer process at the interface of AuNCs@DCTX, and the fluorescence of AuNCs@DCTX was quenched..$^{52,53}$ As expected, the HRTEM image, as shown in Fig. 6A, revealed that the average diameter of particles increased to around $5.02 \mathrm{~nm}$ in the presence of $\mathrm{Cu}^{2+}$ ions (Fig. S7 $\dagger$ ), and the selected area electron diffraction pattern, as shown in Fig. 6B, verified the crystalline nature of the AuNCs@DCTX-Cu ${ }^{2+}$ system. As depicted in Fig. 7A, a distinct peak appeared in the UV-vis absorption spectra of the AuNCs@DCTX-Cu ${ }^{2+}$ system when the concentration of the

Table 1 Comparison of the method proposed in our study with other reported fluorescence nanocluster-based methods for the determination of $\mathrm{Cu}^{2+a}$

\begin{tabular}{|c|c|c|c|c|c|c|}
\hline Probe & Ligands/reductant & $\Phi$ & Linear range $(\mu \mathrm{M})$ & LOD & Sample & References \\
\hline AuNCs@DTT & DTT & $2.09 \%$ & $0-60$ & $80 \mathrm{nM}$ & Human serum & 38 \\
\hline Met-AuNC & Methionine & $2.8 \%$ & $0.05-8$ & $7.9 \mathrm{nM}$ & Soil & 39 \\
\hline GS-Au NCs & GSH & - & $0.100-6.25$ & $86 \mathrm{nM}$ & - & 45 \\
\hline AuNCs & Riboflavin/GSH & - & $0-30$ & $0.9 \mu \mathrm{M}$ & - & 46 \\
\hline AuNCs & DNA/HEPES & - & $0.01-10$ & $10 \mathrm{nM}$ & Human serum, water & 47 \\
\hline hPEI-Cu NCs & hPEI/AA & $7.9 \%$ & $0.022-8.8$ & $8.9 \mathrm{nM}$ & Water & 50 \\
\hline AuNCs@DCTX & DCTX & $11.8 \%$ & $0.01-40$ & $8 \mathrm{nM}$ & CHMs & This work \\
\hline
\end{tabular}

${ }^{a}$ DTT, dithiothreitol; BSA, bovine serum albumin; GSH, L-glutathione; HEPES, 4-(2-hydroxyethyl)-1-piperazineethanesulfonic acid; hPEI, hyperbranched polyethyleneimine; AA, ascorbic acid. 

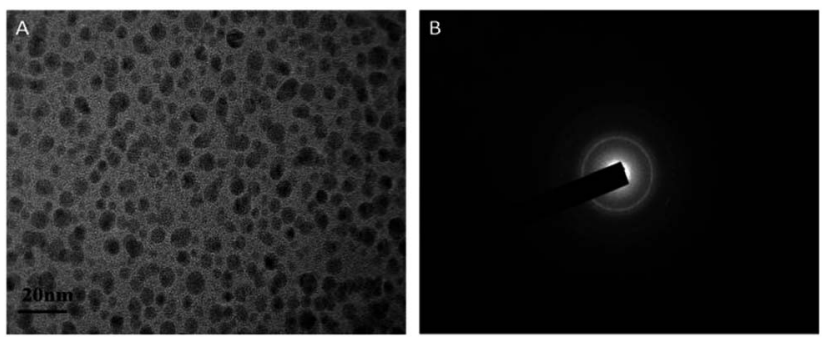

Fig. 6 The HRTEM image (A) and the selected electron diffraction pattern (B) of the prepared AuNCs@DCTX after adding $\mathrm{Cu}^{2+}$ in PBS (scale bar $=20 \mathrm{~nm}$ ).
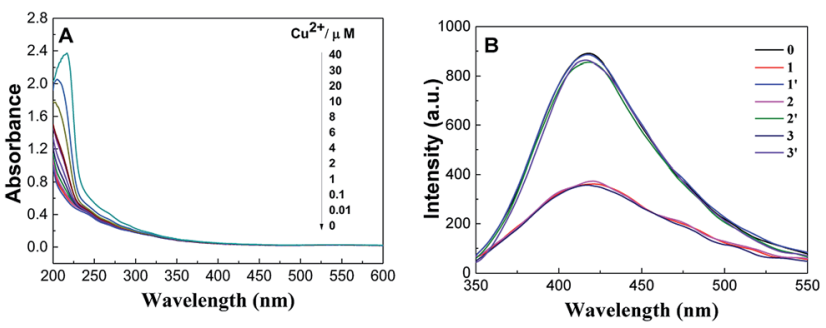

Fig. 7 The UV-Vis absorption spectra (A) of the AuNCs@DCTX after adding $\mathrm{Cu}^{2+}$ with different concentrations in PBS and the fluorescence regeneration results (B) for $\mathrm{Cu}^{2+}$ quenching upon the addition of EDTA in PBS (0: AuNCs@DCTX; 1 : AuNCs@DCTX + Cu ${ }^{2+} ; 1^{\prime}: 1$ + EDTA; $2: 1^{\prime}+$ $\mathrm{Cu}^{2+} ; 2^{\prime}: 2$ + EDTA; $3: 2^{\prime}+\mathrm{Cu}^{2+}$; and $3^{\prime}: 3+$ EDTA).

quenching $\mathrm{Cu}^{2+}$ was above $10 \mu \mathrm{M}$. Furthermore, the absorption peak was red-shifted with an increase in the concentration of $\mathrm{Cu}^{2+}$. This could be another evidence of the coordination between $\mathrm{Cu}^{2+}$ and AuNCs@DCTX. However, the absence of a surface plasmon absorption peak at around $520 \mathrm{~nm}$ further ruled out the possibility of gold nanoparticle formation. To further elucidate the sensing mechanism, a strong chelator, EDTA, was added to the system to compete with AuNCs@DCTX for $\mathrm{Cu}^{2+}$. Fig. 7B shows that after quenching by $\mathrm{Cu}^{2+}$, the fluorescence intensity of AuNCs@DCTX can be reversibly recovered after adding EDTA at $[\mathrm{EDTA}] \approx\left[\mathrm{Cu}^{2+}\right]$. Preliminary experiments indicated that EDTA had no influence on the fluorescence intensity of AuNCs@DCTX in the absence of $\mathrm{Cu}^{2+}$ under the same conditions. The recovery of the fluorescence of AuNCs@DCTX stemmed from the formation of the $\mathrm{Cu}^{2+}$-EDTA complex, allowing the release of AuNCs@DCTX. The reversibility further confirmed that AuNCs@DCTX aggregation was induced by complexation between $\mathrm{Cu}^{2+}$ and the functional groups of DCTX of AuNCs@DCTX.44 Moreover, after three cycles, the fluorescence intensity of AuNCs@DCTX was still maintained at $96.3 \%$ of the initial value; this indicated that the as-prepared AuNCs@DCTX as a probe could be reusable.

To further explore the fluorescence quenching mechanism, the quenching process was fitted by the Stern-Volmer equation: ${ }^{54}$

$$
\frac{F_{0}}{F}=1+K_{\mathrm{SV}}[\mathrm{Q}]
$$

where $K_{\mathrm{SV}}$ is the Stern-Volmer constant, [Q] is the concentration of $\mathrm{Cu}^{2+}$, and $F_{0}$ and $F$ are the fluorescence intensities in the absence and presence of $\mathrm{Cu}^{2+}$, respectively. According to the linear equation shown in Fig. 4B, the Stern-Volmer constant $K_{\mathrm{SV}}$ was $6.94 \times 10^{4} \mathrm{M}^{-1}$. The relatively large magnitude value of $K_{\mathrm{SV}}$ suggested that the fluorescence quenching of AuNCs@DCTX by $\mathrm{Cu}^{2+}$ could be mainly attributed to a static quenching mechanism based on the complexation between $\mathrm{Cu}^{2+}$ ions and AuNCs@DCTX. Moreover, the quenching rate constant $K_{\mathrm{q}}$ can be measured by the dimolecular quenching equation: $:^{55}$

$$
K_{\mathrm{SV}}=K_{\mathrm{q}} \tau_{0}
$$

where $\tau_{0}$ (usually $10^{-8} \mathrm{~s}$ ) is the fluorescence lifetime of AuNCs@DCTX. Based on the value of $K_{S v}$ and the eqn (2), the calculated $K_{\mathrm{q}}$ was $6.94 \times 10^{12} \mathrm{~L} \mathrm{~mol}^{-1} \mathrm{~s}^{-1}$, which was greater than the maximum diffusion collision quenching constant (2.0 $\times 10^{10} \mathrm{~L} \mathrm{~mol}^{-1} \mathrm{~s}^{-1}$ ); this suggested that the fluorescence of AuNCs@DCTX was quenched by $\mathrm{Cu}^{2+}$ through static quenching mechanisms. ${ }^{56}$

\subsection{Determination of $\mathrm{Cu}^{2+}$ in CHMs}

The practicality of AuNCs@DCTX was investigated by determining $\mathrm{Cu}^{2+}$ in four CHMs i.e. licorice, alisma, salvia and poria. We found that iron in these four CHMs, which was about 5-20 times as abundant as copper, would interfere with the determination of $\mathrm{Cu}^{2+}$. Accordingly, a certain amount of $\mathrm{NaF}$, as a complexing agent for $\mathrm{Fe}^{3+}$, was added to the AuNCs@DCTX$\mathrm{Cu}^{2+}$ system, and the analytical results of $\mathrm{Cu}^{2+}$ in the four CHMs were obtained, as listed in Table S1. $\dagger$ Moreover, these four CHMs were analyzed by the FAAS. As shown in Table S1, $\uparrow$ our results with an absolute value of the relative error less than $8.6 \%$ were similar to those obtained by the reference FAAS method. The consistent analytical results confirmed the reliability of the AuNCs@DCTX-based method for the determination of $\mathrm{Cu}^{2+}$ in CHM samples.

\section{Conclusions}

Herein, we successfully prepared water-soluble AuNCs@DCTX via a one-pot approach using the degradation product of CTX as the capping and reducing agent. The synthesis process was simple and environmentally friendly. Due to the high colloidal stability and multifunctional groups, the AuNCs@DCTX probe had acceptable selectivity for the detection of $\mathrm{Cu}^{2+}$ through aggregation-induced fluorescence quenching. Further applications were confirmed by the analysis of licorice, alisma, salvia and poria in the presence of $\mathrm{NaF}$, and the results obtained via our study were in agreement with those determined by the FAAS method; this demonstrated that the proposed probe had great potential for the determination of $\mathrm{Cu}^{2+}$ in complicated samples.

\section{Conflicts of interest}

There are no conflicts to declare. 


\section{Acknowledgements}

The financial support provided by the National Natural Science Foundation of China (No. 21107022), the Key Scientific Research Project of Higher Education of Henan Province of China (No. 18A150034) and the Natural Science Foundation of Henan province of China (No. 182300410257) is gratefully acknowledged.

\section{Notes and references}

1 H. Huo, Y. Liu, W. Liu, J. Sun, Q. Zhang, Y. Zhao, J. Zheng, P. Tu, Y. Song and J. Li, J. Chromatogr. A, 2018, 1558, 37-49.

2 F. Meng, Y. Wei, H. Lu, X. Zhou, J. Liu, G. Li and J. Hou, Chin. Chem. Lett., 2013, 24, 506-508.

3 A. Ting, Y. Chow and W. Tan, J. Tradit. Chin. Med., 2013, 33, 119-124.

4 Z. M. Memon, E. Yilmaz and M. Soylak, Talanta, 2017, 174, 645-651.

5 C. Liu, J. Qin, X. Dou, M. Yang and X. Sun, Chin. Herb. Med., 2018, 10, 117-136.

6 M. T. F. Teodoro, F. d. S. Dias, D. G. da Silva, M. A. Bezerra, A. F. Dantas, L. S. G. Teixeira and A. L. C. Pereira, Microchem. J., 2017, 132, 351-357.

7 G. P. C. Rao, K. Seshaiah, Y. K. Rao and M. C. Wang, J. Agric. Food Chem., 2006, 54, 2868-2872.

8 M. A. Deshmukh, H. K. Patil, G. A. Bodkhe, M. Yasuzawa, P. Koinkar, A. Ramanaviciene, M. D. Shirsat and A. Ramanavicius, Sens. Actuators, B, 2018, 260, 331-338.

9 S. Zhan, H. Xu, W. Zhang, X. Zhan, Y. Wu, L. Wang and P. Zhou, Talanta, 2015, 142, 176-182.

10 T. Sun, Y. Li, Q. Niu, T. Li and Y. Liu, Spectrochim. Acta, Part A, 2018, 195, 142-147.

11 J. R. Bhamore, S. Jha, T. J. Park and S. K. Kailasa, Sens. Actuators, B, 2018, 277, 47-54.

12 J. Wang, J. Yu, X. Wang, L. Wang, B. Li, D. Shen, Q. Kang and L. Chen, Microchim. Acta, 2018, 185, 420.

13 N. Wang, Y. Liu, Y. Li, Q. Liu and M. Xie, Sens. Actuators, B, 2018, 255, 78-86.

14 J. Qi, B. Li, X. Wang, Z. Zhang, Z. Wang, J. Han and L. Chen, Sens. Actuators, B, 2017, 251, 224-233.

15 M. H. Ibrahim, W. Gao, M. Saqib, S. A. Kitte, F. Wu and G. Xu, Biosens. Bioelectron., 2017, 95, 8-14.

16 W. Ding, L. Guan, J. Han, R. Mangala and Z. Luo, Sens. Actuators, B, 2017, 250, 364-371.

$17 \mathrm{X} . \mathrm{Hu}, \mathrm{X} . \mathrm{Mao}, \mathrm{X}$. Zhang and Y. Huang, Sens. Actuators, B, 2017, 247, 312-318.

$18 \mathrm{X} . \mathrm{Hu}, \mathrm{T}$. Liu, Y. Zhuang, W. Wang, Y. Li, W. Fan and Y. Huang, TrAC, Trends Anal. Chem., 2016, 77, 66-75.

19 L. Zhang and E. Wang, Nano Today, 2014, 9, 132-157.

$20 \mathrm{X}$. Wu, Z. Zhang, J. Li, H. You, Y. Li and L. Chen, Sens. Actuators, B, 2015, 211, 507-514.

21 Y. Ding, S. Wang, J. Li and L. Chen, TrAC, Trends Anal. Chem., 2016, 82, 175.

22 L. Chen, C. Wang, Z. Yuan and H. Chang, Anal. Chem., 2014, 87, 216-229.
23 M. Luo, J. Di, L. Li, Y. Tu and J. Yan, Talanta, 2018, 187, 231236.

24 W. Liu, X. Wang, Y. Wang, J. Li, D. Shen, Q. Kang and L. Chen, Sens. Actuators, B, 2018, 262, 810-817.

25 P. Chen, A. P. Periasamy, S. G. Harroun, W. Wu and H. Chang, Coord. Chem. Rev., 2016, 320-321, 129-138.

26 J. Jiang, P. Gao, Y. Zhang, G. Zhang, Y. Zhou, C. Dong and S. Shuang, Talanta, 2017, 174, 44-51.

27 D. Zhao, C. Chen, J. Zhao, J. Sun and X. Yang, Sens. Actuators, $B$, 2017, 247, 392-399.

28 G. Ding, Y. Liu, R. Cong and J. Wang, Talanta, 2004, 62, 9971003.

29 H. Jiang, Y. Li, M. Pelzer, M. J. Cannon, C. Randlett, H. Junga, X. Jiang and Q. C. Ji, J. Chromatogr. A, 2008, 1192, 230-238.

30 M. Yu, Z. Zhu, H. Wang, L. Li, F. Fu, Y. Song and E. Song, Biosens. Bioelectron., 2017, 91, 143-148.

31 A. Bagheri Gh, A. Yosefi rad, M. Rezvani and S. Roshanzamir, Spectrochim. Acta, Part A, 2012, 89, 317-321.

32 L. Wu, Y. Zhang, W. Zhao and Q. Li, J. Chin. Chem. Soc., 2008, 55, 550-556.

33 J. Liu, S. Dong and L. Han, Chin. J. Spectrosc. Lab., 2007, 24, 31-33.

34 H. Deng, F. Wang, X. Shi, H. Peng, A. Liu, X. Xia and W. Chen, Biosens. Bioelectron., 2016, 83, 1-8.

35 H. Huang, H. Li, J. Feng, H. Feng, A. Wang and Z. Qian, Sens. Actuators, B, 2017, 241, 292-297.

36 H. Liu, M. Li, Y. Xia and X. Ren, ACS Appl. Mater. Interfaces, 2017, 9, 120-126.

37 S. Xu, H. Yang, K. Zhao, J. Li, L. Mei, Y. Xie and A. Deng, RSC Adv., 2015, 5, 11343-11348.

38 H. Ding, C. Liang, K. Sun, H. Wang, J. K. Hiltunen, Z. Chen and J. Shen, Biosens. Bioelectron., 2014, 59, 216-220.

39 H. Deng, L. Zhang, S. He, A. Liu, G. Li, X. Lin, X. Xia and W. Chen, Biosens. Bioelectron., 2015, 65, 397-403.

40 Z. Wu and R. Jin, Nano Lett., 2010, 10, 2568-2573.

41 L. Tian, W. Zhao, L. Li, Y. Tong, G. Peng and Y. Li, Sens. Actuators, B, 2017, 240, 114-124.

42 S. Xu, X. Feng, T. Gao, R. Wang, Y. Mao, J. Lin, X. Yu and X. Luo, Anal. Chim. Acta, 2017, 958, 22-29.

43 X. Yang, Z. Jia, Z. Tan, H. Xu, N. Luo and X. Liao, Food Control, 2016, 70, 286-292.

44 C. V. Durgadas, C. P. Sharmaa and K. Sreenivasan, Analyst, 2011, 136, 933-940.

45 G. Zhang, Y. Li, J. Xu, C. Zhang, S. Shuang, C. Dong and M. M. F. Choi, Sens. Actuators, B, 2013, 183, 583-588.

46 M. Zhang, H. Le, X. Jiang, S. Guo, H. Yu and B. Ye, Talanta, 2013, 117, 399-404.

47 T. Qing, H. Bu, X. He, D. He, B. Zhou, H. Sun, R. Jia, W. Ma and K. Wang, Anal. Methods, 2017, 9, 6222-6227.

48 K. Shanmugaraj and M. Ilanchelian, RSC Adv., 2016, 6, 54518-54524.

49 S. Chen, Y. Kuang, P. Zhang, Y. Huang, A. Wen, X. Zeng, R. Feng, H. Nie, X. Jiang and Y. Long, Sens. Actuators, B, 2017, 253, 283-291.

50 Z. Liu, J. Qi, C. Hu, L. Zhang, W. Song, R. Liang and J. Qiu, Anal. Chim. Acta, 2015, 895, 95-103. 
51 S. Li, J. Feng, P. Huang and F. Wu, New J. Chem., 2017, 41, 12930-12936.

52 J. S. Anjali Devi, S. Salini, A. H. Anulekshmi, G. L. Praveen and G. Sony, Sens. Actuators, B, 2017, 246, 943-951.

53 W. Chen, X. Tu and X. Guo, Chem. Commun., 2009, 17361738.
54 H. Jia, C. Feng and C. Tian, Anal. Sci., 2018, 34, 1079-1083. 55 M. Mehrzad-Samarin, F. Faridbod and M. R. Ganjali, Spectrochim. Acta, Part A, 2019, 206, 430-436.

56 D. Wu, Z. Chen, G. Huang and X. Liu, Sens. Actuators, A, 2014, 205, 72-78. 